\title{
Particle Propagator in Elementary Quantum Mechanics: a New Path Integral Derivation
}

\author{
Stefano Ansoldi†, Antonio Aurilia抑 and Euro Spallucci何 \\ $\dagger$ Dipartimento di Fisica Teorica, Università di Trieste, \\ Strada Costiera 11, 34014 Trieste, \\ INFN, Sezione di Trieste \\ $\ddagger$ Department of Physics, California State Polytechnic University, Pomona, CA 91768
}

\begin{abstract}
This paper suggests a new way to compute the path integral for simple quantum mechanical systems. The new algorithm originated from previous research in string theory. However, its essential simplicity is best illustrated in the case of a free non relativistic particle, discussed here, and can be appreciated by most students taking an introductory course in Quantum Mechanics. Indeed, the emphasis is on the role played by the entire family of classical trajectories in terms of which the path integral is computed exactly using a functional representation of the Dirac delta-distribution. We argue that the new algorithm leads to a deeper insight into the connection between classical and quantum systems, especially those encountered in high energy physics.
\end{abstract}

PACS numbers:

Submitted to: Europ. J. Phys.

$\S$ E-mail address: ANSOLDI@TRIESTE.INFN.IT

|| E-mail address: AAURILIA@CSUPOMONA.EDU

ๆ E-mail address: SPALLUCCI@TRIESTE.INFN.IT 


\section{Introduction}

It is often argued that a formulation of quantum mechanics in terms of path integrals is too advanced to lie within the scope of most undergraduate courses. On the other hand, a great deal of physics is done, nowadays, using Feynman's path integral method, with applications ranging from gauge theories in high energy physics to solid state physics, and statistical mechanics. As a result, the amount of pedagogical literature dedicated to a discussion of the path integral approach to quantum mechanics is increasing steadily [1, 2, 3, 4, 5].

Quantum mechanics is probably the most challenging paradigm of physics that a student will ever come across, and the pedagogical effectiveness of Feynman's method, beautifully expounded by the author himself in his original work [6], lies in its appeal to intuition while addressing the most fundamental principles of the theory. Indeed, it seems to us that one of the major achievements of Feynman's formulation of quantum mechanics was to restore the particle trajectory concept at the quantum level. Our purpose, then, is to suggests a specific algorithm that emphasizes the privileged role that classical trajectories play in constructing the "sum over histories" envisaged by Feynman in order to describe the quantum evolution of a particle.

This paper is an outgrowth of an earlier investigation in string theory where the computational method described here was first applied [7, 8, 9]. However, in order to illustrate the method in the simplest context, we apply it to the familiar case of a free, non relativistic particle. We show that the path integral can be computed exactly by summing over the whole family of trajectories which are solutions of the classical equations of motion. This is in contrast to the conventional approach to the path integral in which Newtonian dynamics singles out a unique classical trajectory which is then perturbed by quantum fluctuations. The main ideas and techniques are discussed in the case of a free particle with the bare minimum of formal apparatus which should be within grasp of most undergraduate physics majors taking a first course in quantum mechanics.

Hopefully, the applicability of our approach to higher dimensional objects will justify the claim, made in the abstract, that our method applies to "simple quantum mechanical systems" besides the point particle case considered in this paper.

In order to place our work in the right perspective, we begin Section 2 with a brief overview of the discretization procedure that forms the basis of the "sum over histories" approach to Quantum Mechanics. This will give us the opportunity to single out the precise point of departure of our own approach from the conventional one based on the "Lagrangian path integral".

In Section 3 we construct the propagation kernel and make contact with the diffusion equation of a free, non relativistic particle.

In Appendix A we outline a possible extension of our definition of path integral to the case of a particle in an external potential.

Finally, Appendix B is a compendium of three basic formulae which are especially 
relevant to the discussion in the text.

\section{Some Remarks about Path Integration}

In this section we review briefly the definition of phase space path integral. The main purpose here is twofold: first, we wish to present a self-contained exposition for those readers who may not be acquainted with the idea of "sum over histories" proposed by Feynman [6, 10]; second, we wish to isolate those fundamental properties of Quantum Mechanics, encoded in the path integral approach, which are relevant to the subsequent discussion of our own computational method .

One such fundamental property is the distinction between the square of the absolute value of the wave function, or probability density, and the wave function itself, or probability amplitude. With that distinction in mind, the peculiarity of the quantum mechanical world is that, in contrast to the classical rules of conditional probability, one computes probability amplitudes for the paths, and then sum the amplitudes; when amplitudes are superimposed, and then squared, an interference pattern is induced in the probability density. The famed "particle--wave duality" of the quantum world stems essentially from that new computational rule. According to Dirac and Feynman [11, the basic difference between classical and quantum mechanics is that the former selects, through the stationary action principle, a single trajectory connecting the initial and final particle position, while the latter assumes that a particle "moves simultaneously along all possible trajectories" connecting the initial and final end points. Of course, not all trajectories are equal, even if they correspond to wave functions having the same absolute value: the action $S[\overrightarrow{\boldsymbol{y}}(t)]$ of a particular path connecting the two fixed end points determines the phase of the propagation amplitude associated with that path. The phase factor corresponding to any individual path can be written in the form $\exp \{i S[\overrightarrow{\boldsymbol{y}}(t), \overrightarrow{\boldsymbol{p}}(t)] / \hbar\}$, and a very effective way to describe the quantum mechanical behavior of a particle is through the sum over histories proposed by Feynman. Symbolically, the amplitude to evolve from $\overrightarrow{\boldsymbol{x}}_{0}$ to $\overrightarrow{\boldsymbol{x}}$ is

$$
A\left(\overrightarrow{\boldsymbol{x}}_{0}, \overrightarrow{\boldsymbol{x}}\right)=\sum_{\text {paths from } \overrightarrow{\boldsymbol{x}}_{0} \text { to } \overrightarrow{\boldsymbol{x}}} \Phi[\overrightarrow{\boldsymbol{y}}(t), \overrightarrow{\boldsymbol{p}}(t)],
$$

where

$$
\Phi[\overrightarrow{\boldsymbol{y}}(t), \overrightarrow{\boldsymbol{p}}(t)]=\mathcal{K} \cdot \exp \left\{\frac{i}{\hbar} S[\overrightarrow{\boldsymbol{y}}(t), \overrightarrow{\boldsymbol{p}}(t)]\right\} .
$$

A sum of this type, defined over a space of functions, i.e., $\overrightarrow{\boldsymbol{y}}(t)$, and $\overrightarrow{\boldsymbol{p}}(t)$, is a functional integral, and the basic problem of the Feynman formulation of quantum mechanics is to determine what that formal sum means.

In order to construct the sum over all paths in phase space, one may follow the evolution of the system in a total time interval $\left[t, t^{\prime}\right]$ by dividing such interval in $N+1$ subintervals, with end points labeled by $t_{1}, t_{2}, \ldots, t_{i}, \ldots, t_{N+1}$ so that

$$
\begin{aligned}
& t=t_{0} \\
& t^{\prime}=t_{N+1} .
\end{aligned}
$$


For convenience, one may also choose each of the above intervals to be of equal length $\epsilon$,

$$
t_{i+1}-t_{i}=\epsilon \quad
$$

in such a way that

$$
t_{k}=t+k \epsilon \quad, \quad k=0, \ldots, N+1 .
$$

Next, one has to determine an equation for the partial amplitude

$$
A\left(q_{i+1}, t_{i+1} ; q_{i}, t_{i}\right) \equiv\left\langle q_{i+1}, t_{i+1} \mid q_{i}, t_{i}\right\rangle
$$

that the system evolves from a position with coordinate $q_{i}$ at time $t_{i}$ to a position with coordinate $q_{i+1}$ at time $t_{i+1}$. Using the bracket notation, the amplitude for the first subinterval takes the form

$$
\begin{aligned}
\langle\tilde{q}, \epsilon \mid \bar{q}, 0\rangle & =\langle\tilde{q}|\exp (-i H \epsilon)| \bar{q}\rangle \\
& =\delta(\tilde{q}-\bar{q})-i \epsilon\langle\tilde{q}|H| \bar{q}\rangle+\mathcal{O}\left(\epsilon^{2}\right) \\
& =\int \frac{d p}{2 \pi} e^{i p(\tilde{q}-\bar{q})}-\int \frac{d p}{2 \pi} e^{i p(\tilde{q}-\bar{q})} i \epsilon H\left(p, \frac{\tilde{q}+\bar{q}}{2}\right)+\mathcal{O}\left(\epsilon^{2}\right) \\
& =\int \frac{d p}{2 \pi} e^{i p(\tilde{q}-\bar{q})}\left[1-i \epsilon H\left(p, \frac{\tilde{q}+\bar{q}}{2}\right)\right]+\mathcal{O}\left(\epsilon^{2}\right) \\
& =\int \frac{d p}{2 \pi} \exp \left\{i\left[p(\tilde{q}-\bar{q})-\epsilon H\left(p, \frac{\tilde{q}+\bar{q}}{2}\right)\right]\right\}
\end{aligned}
$$

Generalizing the above expression to a generic subinterval, we find

$$
\left\langle q_{i+1}, t_{i+1} \mid q_{i}, t_{i}\right\rangle=\int \frac{d p_{i}}{2 \pi} \exp \left\{i\left[p_{i}\left(q_{i+1}-q_{i}\right)-H\left(p_{i}, \frac{q_{i+1}+q_{i}}{2}\right)\left(t_{i+1}-t_{i}\right)\right]\right\} .
$$

Approximating the path from $q$ at time $t$ to $q^{\prime}$ at time $t^{\prime}$ with a finer subdivision of the total time interval, and in view of the fact that there are no predetermined conditions on the coordinate variable at intermediate times, the total amplitude can be written as the product of the amplitudes associated with each subinterval, integrated over all possible intermediate positions:

$$
A\left(q^{\prime}, t^{\prime} ; q, t\right)=\lim _{N \rightarrow+\infty} \int d q_{1} \int d q_{2} \ldots \int d q_{N} \prod_{i}^{1, N+1}\left\langle q_{i}, t_{i} ; q_{i-1}, t_{i-1}\right\rangle
$$

Combining the two equations above, we are led to the final step of the discretization procedure

$$
\begin{gathered}
A\left(q^{\prime}, t^{\prime} ; q, t\right)=\lim _{N \rightarrow+\infty} \int d q_{1} \int d q_{2} \int \ldots \int d q_{N} \cdot \\
\cdot \int \frac{d p_{1}}{2 \pi} \int \frac{d p_{2}}{2 \pi} \int \ldots \int \frac{d p_{N+1}}{2 \pi} \cdot \\
\cdot e^{i\left[p_{1}\left(q_{1}-q_{0}\right)-\left(t_{1}-t_{0}\right) H\left(p_{1}, \frac{q_{1}+q_{0}}{2}\right)\right]} \cdot \\
\cdot e^{i\left[p_{2}\left(q_{2}-q_{1}\right)-\left(t_{2}-t_{1}\right) H\left(p_{2}, \frac{q_{2}+q_{1}}{2}\right)\right]} \cdot \ldots \cdot \\
\cdot e^{i\left[p_{N+1}\left(q_{N+1}-q_{N}\right)-\left(t_{N+1}-t_{N}\right) H\left(p_{N+1}, \frac{q_{N+1}+q_{N}}{2}\right)\right]}
\end{gathered}
$$




$$
\begin{aligned}
=\lim _{N \rightarrow+\infty} & \left(\prod_{i}^{1, N} \int d q_{i}\right)\left(\prod_{j}^{1, N+1} \int d p_{j}\right) . \\
\cdot & e^{i\left\{\sum_{k}^{1, N+1}\left[p_{k}\left(q_{k}-q_{k-1}\right)-\left(t_{k}-t_{k-1}\right) H\left(p_{k}, \frac{q_{k}+q_{k-1}}{2}\right)\right]\right\}} \\
=\lim _{N \rightarrow+\infty} & \left(\prod_{i}^{1, N} \int d q_{i}\right)\left(\prod_{j}^{1, N+1} \int d p_{j}\right) . \\
\cdot & e^{i\left\{\sum_{k}^{1, N+1}\left[p_{k} \frac{q_{k}-q_{k-1}}{t_{k}-t_{k-1}}-H\left(p_{k}, \frac{q_{k}+q_{k-1}}{2}\right)\right]\left(t_{k}-t_{k-1}\right)\right\}} .
\end{aligned}
$$

The expression in the last equality represents the discrete version of the path integral in phase space

$$
\int[\mathcal{D} q] \int[\mathcal{D} p] \exp \{i[p \dot{q}-H(p, q)] d t\} .
$$

which is the starting point for computing the sum over histories for the majority of the integrable systems encountered in the literature. Equation (9) is also the starting point of our own approach. However, in terms of the discretization procedure outlined above, a further simple elaboration of the phase space path integral leads to a computational method which seems mathematically more efficient and physically more enlightening, at least for some complex systems encountered in high energy physics.

Consider the first term in the expression (9), namely

$$
\int p \dot{q} d t=\int p d q
$$

This term corresponds to the discrete sum

$$
\sum_{k}^{1, N+1} p_{k}\left(q_{k}-q_{k-1}\right)
$$

which we can rewrite as follows:

$$
\begin{aligned}
& \sum_{k}^{1, N+1} p_{k}\left(q_{k}-q_{k-1}\right)= \\
= & \sum_{k}^{1, N+1} p_{k} q_{k}-\sum_{k}^{1, N+1} p_{k} q_{k-1} \\
= & \sum_{k}^{1, N+1} p_{k} q_{k}-\sum_{k}^{1, N+1} p_{k} q_{k-1}-\sum_{k}^{1, N+1} p_{k-1} q_{k-1}+\sum_{k}^{1, N+1} p_{k-1} q_{k-1} \\
= & \sum_{k}^{1, N+1}\left(p_{k} q_{k}-p_{k-1} q_{k-1}\right)-\sum_{k}^{1, N+1}\left(p_{k}-p_{k-1}\right) q_{k-1} \\
= & p_{N+1} q_{N+1}-p_{0} q_{0}-\sum_{k}^{1, N}\left(p_{k}-p_{k-1}\right) q_{k-1} .
\end{aligned}
$$


The first two terms in the above expression represent a boundary contribution to the path integral, whereas the remaining sum corresponds to the following integral

$$
\sum_{i}^{1, N}\left(p_{k}-p_{k-1}\right) q_{k-1} \Longrightarrow \int q d p=\int q \dot{p} d t
$$

Next, in what follows we make use of the following equality

$$
\int d q_{k-1} \exp \left\{i\left(p_{k}-p_{k-1}\right) q_{k-1}\right\}=\delta\left(p_{k}-p_{k-1}\right)
$$

which gives the well known representation of the Dirac delta function as Fourier transform of the imaginary exponential. Thus, the net result of the above rearrangement of terms is encoded in the following correspondence

$$
\int[\mathcal{D} q] \exp \left\{i \int d t q \dot{p}\right\}=\delta[\dot{p}] \quad \Longrightarrow \quad \lim _{N \rightarrow \infty} \mathcal{N}\left(t_{j}\right) \prod_{i}^{1, N} \delta\left(\frac{p_{k}-p_{k-1}}{t_{k}-t_{k-1}}\right) \quad
$$

which we take as a definition of the "functional Dirac delta distribution".

In the next section, we apply this mathematical rearrangement of terms in the phase space path integral to compute the propagation kernel of a non relativistic particle. As we shall see, the physical payoff is a novel interpretation of the sum over histories in the sense that it underscores the special role played by the entire family of trajectories which are solutions of the classical equations of motion.

\section{Non-Relativistic Particle}

\subsection{Path Integral and Propagation Kernel}

The classical dynamics of a non-relativistic point-particle with mass $m$ is encoded into the Lagrange function, or in its corresponding Hamiltonian

$$
\begin{aligned}
& L(\overrightarrow{\boldsymbol{y}}(t), \dot{\overrightarrow{\boldsymbol{y}}}(t) ; t)=\frac{1}{2} m \dot{\overrightarrow{\boldsymbol{y}}}^{2} \\
& H(\overrightarrow{\boldsymbol{y}}(t), \overrightarrow{\boldsymbol{p}}(t) ; t)=\frac{1}{2 m} \overrightarrow{\boldsymbol{p}}^{2} .
\end{aligned}
$$

¿From here follow the classical equations of motion:

$$
\begin{aligned}
& \frac{d \overrightarrow{\boldsymbol{p}}}{d t}=0 \quad \Rightarrow \quad \overrightarrow{\boldsymbol{p}}=\text { cost. } \equiv \overrightarrow{\boldsymbol{q}} \\
& \frac{d H}{d t}=0 \quad \Rightarrow \quad H=\text { cost. } \equiv E \\
& m \frac{d \overrightarrow{\boldsymbol{y}}}{d t}=\overrightarrow{\boldsymbol{q}} \quad \Rightarrow \quad \overrightarrow{\boldsymbol{y}}(t)=\frac{1}{m} \overrightarrow{\boldsymbol{q}} t+\overrightarrow{\boldsymbol{x}}_{0},
\end{aligned}
$$

where we have taken into account the boundary conditions

$$
\overrightarrow{\boldsymbol{y}}(0)=\overrightarrow{\boldsymbol{x}}_{0} \quad, \quad \overrightarrow{\boldsymbol{y}}(T)=\overrightarrow{\boldsymbol{x}} \quad \Rightarrow \quad \overrightarrow{\boldsymbol{q}}=m \frac{\overrightarrow{\boldsymbol{x}}-\overrightarrow{\boldsymbol{x}}_{0}}{T}
$$

so that

$$
\overrightarrow{\boldsymbol{y}}(t)=\frac{\overrightarrow{\boldsymbol{x}}-\overrightarrow{\boldsymbol{x}}_{0}}{T} t+\overrightarrow{\boldsymbol{x}}_{0}
$$


Furthermore, for later reference, we recall that the classical action

$$
\begin{aligned}
S_{\text {cl. }}\left(\overrightarrow{\boldsymbol{x}}, \overrightarrow{\boldsymbol{x}}_{0} ; T\right) & =\frac{1}{2} m \int_{0}^{T} d t\left(\frac{\overrightarrow{\boldsymbol{x}}-\overrightarrow{\boldsymbol{x}}_{0}}{T}\right)^{2} \\
& =\frac{m}{2 T}\left|\overrightarrow{\boldsymbol{x}}-\overrightarrow{\boldsymbol{x}}_{0}\right|^{2}
\end{aligned}
$$

is a solution of the Jacobi equation

$$
\frac{\partial S_{\mathrm{cl} .}}{\partial T}+\frac{1}{2 m} \vec{\nabla} S_{\mathrm{cl} .} \cdot \vec{\nabla} S_{\mathrm{cl} .}=0
$$

Equivalently, one can follow the particle evolution by determining the propagation amplitude $K\left(\overrightarrow{\boldsymbol{x}}-\overrightarrow{\boldsymbol{x}}_{0} ; T\right)$ that a particle propagates from an initial position $\overrightarrow{\boldsymbol{x}}_{0}$ to a final position $\overrightarrow{\boldsymbol{x}}$. As we have shown in the previous section, the amplitude is given by a formal sum over all phase space paths connecting $\overrightarrow{\boldsymbol{x}}_{0}$ to $\overrightarrow{\boldsymbol{x}}$ in a total time $T$, each path carrying a weight given by the phase factor $\exp (i S[$ path $] / \hbar)$. We have also reviewed the standard method that gives meaning to the sum over histories: it goes through a discretization procedure which consists in subdividing the total time lapse $T$ into a number of infinitesimal intervals, thereby approximating the smooth phase space trajectory followed by a classical particle with a succession of "jagged" paths. It seems worth emphasizing at this point, that in the above implementation of the path integral, the classical trajectory followed by a particle is uniquely specified by Newtonian mechanics. Furthermore, we hasten to say that the sum over histories, so defined, is a sum over trajectories which are nowhere differentiable. As a matter of fact, a post modern interpretation of Feynman's discussion is that the quantum mechanical path of a particle is inherently fracta円. The gist of the argument is that, when a particle is more and more precisely located in space, its trajectory becomes more and more erratic as a consequence of Heisenberg's principle. In other words, it is the addition of quantum fluctuations around the classical trajectory that gives meaning to the idea that "a particle moves along all possible paths" connecting the initial and final configurations. Be that as it may, the discretization procedure turns the functional integral into an infinite product of ordinary integrals [13]. For some elementary systems, the integration over the momenta can be carried out, albeit with some efforts, and the final result for the amplitude is the "Lagrangian path integral":

$$
K\left(\overrightarrow{\boldsymbol{x}}, \overrightarrow{\boldsymbol{x}}_{0} ; T\right)=\int_{\overrightarrow{\boldsymbol{x}}_{0}}^{\overrightarrow{\boldsymbol{x}}}[\mathcal{D} y(t)] \exp \frac{i}{\hbar} \int_{0}^{T} L[\overrightarrow{\boldsymbol{y}}(t), \dot{\overrightarrow{\boldsymbol{y}}}(t) ; t] d t .
$$

For more complex systems, such as the relativistic extended systems encountered in contemporary high energy physics, the expression (24), may not tell the full story, and we have found it advantageous to start with a sum over histories in phase space. Thus, in order to illustrate our computational method, we start directly from the canonical

+ This interpretation, clearly implied in the book by Feynman and Hibbs 10, was revisited many years ago by Abbott and Wise 12, and reviewed by the authors in the larger, but mostly unexplored context of the microscopic structure of quantum spacetime [4, 8]. 
phase space path integral for a free, non-relativistic particle

$K\left(\overrightarrow{\boldsymbol{x}}, \overrightarrow{\boldsymbol{x}}_{0} ; T\right)=N \int_{\overrightarrow{\boldsymbol{x}}_{0}}^{\overrightarrow{\boldsymbol{x}}}[\mathcal{D} y(t)][\mathcal{D} p(t)] \exp \frac{i}{\hbar} \int_{0}^{T} d t\left[\overrightarrow{\boldsymbol{p}}(t) \cdot \dot{\overrightarrow{\boldsymbol{y}}}(t)-H_{0}(\overrightarrow{\boldsymbol{p}})\right]$,

where

$$
H_{0}(\overrightarrow{\boldsymbol{p}})=\frac{\overrightarrow{\boldsymbol{p}}^{2}}{2 m}
$$

and $N$ is a normalization constant to be fixed later on.

The focal point of our approach is the recognition that the path integral automatically assigns a special role to the whole family of trajectories which are solutions of the classical equation of motion. The precise meaning of the above statement is illustrated by implementing the computational steps following Eq.(9). Thus, we write the first term in the action as

$$
\int_{0}^{T} d t \overrightarrow{\boldsymbol{p}} \cdot \dot{\overrightarrow{\boldsymbol{y}}}=\int_{0}^{T} d t \frac{d}{d t}[\overrightarrow{\boldsymbol{p}} \cdot \overrightarrow{\boldsymbol{y}}]-\int_{0}^{T} d t \overrightarrow{\boldsymbol{y}} \cdot \dot{\overrightarrow{\boldsymbol{p}}}=\int_{x_{0}}^{x} d(\overrightarrow{\boldsymbol{p}} \cdot \overrightarrow{\boldsymbol{y}})-\int_{0}^{T} d t \overrightarrow{\boldsymbol{y}} \cdot \dot{\overrightarrow{\boldsymbol{p}}} .
$$

Accordingly, the path integral reads

$$
\begin{aligned}
& K\left(\overrightarrow{\boldsymbol{x}}, \overrightarrow{\boldsymbol{x}}_{0} ; T\right)= \\
& \quad=N \int_{\overrightarrow{\boldsymbol{x}}_{0}}^{\overrightarrow{\boldsymbol{x}}}[\mathcal{D} y(t)][\mathcal{D} p(t)] \exp \left\{\frac{i}{\hbar}\left[\int_{\overrightarrow{\boldsymbol{x}}_{0}}^{\overrightarrow{\boldsymbol{x}}} d(\overrightarrow{\boldsymbol{p}} \cdot \overrightarrow{\boldsymbol{y}})-\int_{0}^{T} d t \overrightarrow{\boldsymbol{y}}(t) \cdot \dot{\overrightarrow{\boldsymbol{p}}}(t)-\int_{0}^{T} d t H_{0}(\overrightarrow{\boldsymbol{p}})\right]\right\}
\end{aligned}
$$

and we note that, while the first term in the above expression is the integral of a total differential, and therefore independent of the path connecting the two points $\left(\overrightarrow{\boldsymbol{x}}_{0}, \overrightarrow{\boldsymbol{x}}\right)$, the second term depends linearly on the spatial trajectory $\overrightarrow{\boldsymbol{y}}(t)$. A closer look at this term shows that it is a (functional) Dirac-delta distribution represented as a "Fourier integral" over the functions $\overrightarrow{\boldsymbol{y}}(t)$

$$
\int_{\overrightarrow{\boldsymbol{x}}_{0}}^{\overrightarrow{\boldsymbol{x}}}[\mathcal{D} y(t)] \exp \left\{-\frac{i}{\hbar} \int_{0}^{T} d t \overrightarrow{\boldsymbol{y}}(t) \cdot \dot{\overrightarrow{\boldsymbol{p}}}(t)\right\}=\delta\left[\frac{d \overrightarrow{\boldsymbol{p}}}{d t}\right] .
$$

The Dirac-delta in equation (28) is non-vanishing only when its argument is zero, i.e., when the momentum $\overrightarrow{\boldsymbol{p}}$ solves the classical equation of motion

$$
\frac{d \overrightarrow{\boldsymbol{p}}}{d t}=0 \quad \Rightarrow \quad \overrightarrow{\boldsymbol{p}}(t)=\text { const. } \equiv \overrightarrow{\boldsymbol{q}}
$$

This is our first, and central result which applies equally well, "mutatis mutandis", to relativistic point-particles and relativistic extended objects: once the spatial trajectories $\overrightarrow{\boldsymbol{y}}(t)$ are integrated out, the resulting path integral is non-vanishing only when the surviving integration variable, namely the three-momentum vector along the trajectory, is constrained to satisfy equation (29). This information is encoded in the following expression for the propagation amplitude

$$
\begin{aligned}
& K\left(\overrightarrow{\boldsymbol{x}}, \overrightarrow{\boldsymbol{x}}_{0} ; T\right)= \\
& \quad=N \int[\mathcal{D} p(t)] \delta\left[\frac{d \overrightarrow{\boldsymbol{p}}}{d t}\right] \exp \left[\frac{i}{\hbar} \int_{\overrightarrow{\boldsymbol{x}}_{0}}^{\overrightarrow{\boldsymbol{x}}} d(\overrightarrow{\boldsymbol{p}} \cdot \overrightarrow{\boldsymbol{y}})\right] \exp \left\{-\frac{i}{\hbar} \int_{0}^{T} d t H_{0}(\overrightarrow{\boldsymbol{p}})\right\},
\end{aligned}
$$


where only the restricted family of constant (i.e., time independent) $\overrightarrow{\boldsymbol{p}}$-trajectories contributes to the path integral. Note that the value of the three-momentum along a classical trajectory is a fixed, even though arbitrary, number. Thus, on the mathematical side, the pay-off of our procedure is that we have traded the original path integral, i.e., a functional integral, with a single ordinary integral over the constant, but numerically arbitrary, components of the three-momentum.

On the physical side, our method underscores a conceptual difference between the mechanism of propagation envisaged here and the conventional one. By this we mean that in the conventional interpretation of the path integral, the sum over histories is obtained by summing over all possible quantum fluctuations around a single classical trajectory which is uniquely defined because both extremal position and momentum are preassigned. In contrast, in our interpretation of sum over histories, only the extremal coordinates of the particle are precisely specified by the boundary conditions, whereas the corresponding value of the momentum at the end points is constant but arbitrary, in consistency with the uncertainty principle. Since the momentum is a vector, it follows that all paths in configuration space contribute to the evolution of the wave function. In the above sense, it seems to us that the complementarity between particle and wave behavior and the role of the uncertainty principle are especially evident in our approach. To summarize our discussion so far, "the sum over histories" of a free particle between two fixed end points, can be translated into the integration over all possible values of the linear momentum. Put briefly,

$$
\begin{aligned}
\int[\mathcal{D} p(t)] \delta[d \overrightarrow{\boldsymbol{p}} / d t](\ldots) & =\text { sum over the classical momenta } \\
& =\text { sum over constant momentum trajectories } \\
& =\int d^{3} q(\ldots) \equiv \text { ordinary momentum integral } .
\end{aligned}
$$

Next, if the momentum is constant, then the Hamiltonian is constant as well, and we can write

$$
\int d^{3} q \exp \left[\frac{i}{\hbar} \overrightarrow{\boldsymbol{q}} \cdot \int_{\overrightarrow{\boldsymbol{x}}_{0}}^{\overrightarrow{\boldsymbol{x}}} d \overrightarrow{\boldsymbol{y}}-\frac{i \overrightarrow{\boldsymbol{q}}^{2}}{2 m \hbar} T\right]=\left[\operatorname{det}\left(\frac{i T}{2 \hbar m} \delta_{i j}\right)\right]^{-1 / 2} \exp \left[\frac{i m}{2 \hbar T}\left|\overrightarrow{\boldsymbol{x}}-\overrightarrow{\boldsymbol{x}}_{0}\right|^{2}\right]
$$

where we have used the identity

$$
\int_{\overrightarrow{\boldsymbol{x}}_{0}}^{\overrightarrow{\boldsymbol{x}}} d \overrightarrow{\mathbf{y}}=\overrightarrow{\boldsymbol{x}}-\overrightarrow{\boldsymbol{x}}_{0}
$$

and the formula for the multidimensional Gaussian integral listed in Appendix Appendix B. As a result, we obtain

$$
K\left(\overrightarrow{\boldsymbol{x}}, \overrightarrow{\boldsymbol{x}}_{0} ; T\right)=N\left(\frac{2 \hbar m}{i \pi T}\right)^{3 / 2} \exp \left[\frac{i m}{2 \hbar T}\left|\overrightarrow{\boldsymbol{x}}-\overrightarrow{\boldsymbol{x}}_{0}\right|^{2}\right] .
$$

Finally, the normalization constant $N$ can be fixed by the condition that, in the limit of a vanishing lapse of time, the particle is bound to be in its initial position. In other 
words,

$$
\lim _{T \rightarrow 0} K\left(\overrightarrow{\boldsymbol{x}}-\overrightarrow{\boldsymbol{x}}_{0} ; T\right)=\delta^{3}\left(\overrightarrow{\boldsymbol{x}}-\overrightarrow{\boldsymbol{x}}_{0}\right) \quad \Rightarrow \quad N=\left(\frac{1}{2 \pi \hbar^{2}}\right)^{3 / 2}
$$

Hence,

$$
\begin{aligned}
K\left(\overrightarrow{\boldsymbol{x}}-\overrightarrow{\boldsymbol{x}}_{0} ; T\right) & =\left(\frac{m}{2 i \pi \hbar T}\right)^{3 / 2} \exp \left[\frac{i m}{2 \hbar T}\left|\overrightarrow{\boldsymbol{x}}-\overrightarrow{\boldsymbol{x}}_{0}\right|^{2}\right] \\
& =\left(\frac{m}{2 i \pi \hbar T}\right)^{3 / 2} \exp \left[i S_{\mathrm{cl} .}\left(\overrightarrow{\boldsymbol{x}}-\overrightarrow{\boldsymbol{x}}_{0} ; T\right) / \hbar\right],
\end{aligned}
$$

where the phase factor is just the classical action measured in $\hbar$ units

$$
S_{\text {cl. }}\left(\overrightarrow{\boldsymbol{x}}-\overrightarrow{\boldsymbol{x}}_{0} ; T\right)=\frac{m}{2} \int_{0}^{T} d t\left(\frac{\overrightarrow{\boldsymbol{x}}-\overrightarrow{\boldsymbol{x}}_{0}}{T}\right)^{2}=\frac{m}{2 T}\left|\overrightarrow{\boldsymbol{x}}-\overrightarrow{\boldsymbol{x}}_{0}\right|^{2} .
$$

The above expression for the propagation kernel is a well known result which tests the consistency of our approach: as Feynman and Hibbs demonstrated, for any lagrangian quadratic in the position and velocity variables, the corresponding propagation amplitude is given by a pre-factor, which is a function of the evolution parameter lapse, multiplied by the exponential of "i" times the classical action in $\hbar$ units.

\subsection{Diffusion Equation and Propagation Kernel}

In the previous subsection, we have deduced the form of the propagation kernel (35), by evaluating the sum over histories as a Gaussian integral. With this result in hands, one can make contact with the more familiar formulation of quantum mechanics by showing that the propagation kernel $K$ satisfies a diffusion equation of the Schrödinger type. To see this, first we calculate

$$
\frac{\partial}{\partial T} K\left(\overrightarrow{\boldsymbol{x}}-\overrightarrow{\boldsymbol{x}}_{0} ; T\right)=N \int d^{3} q\left(-i \frac{\overrightarrow{\boldsymbol{q}} \cdot \overrightarrow{\boldsymbol{q}}}{2 \hbar m}\right) \exp \left[\frac{i}{\hbar} \overrightarrow{\boldsymbol{q}} \cdot \int_{\overrightarrow{\boldsymbol{x}}_{0}}^{\overrightarrow{\boldsymbol{x}}} d \overrightarrow{\boldsymbol{y}}\right] \exp \left\{-\frac{i T}{2 \hbar m} \overrightarrow{\boldsymbol{q}} \cdot \overrightarrow{\boldsymbol{q}}\right\},
$$

and

$$
\Delta_{x} K\left(\overrightarrow{\boldsymbol{x}}-\overrightarrow{\boldsymbol{x}}_{0} ; T\right)=N \int d^{3} q\left(-\frac{\overrightarrow{\boldsymbol{q}} \cdot \overrightarrow{\boldsymbol{q}}}{\hbar^{2}}\right) \exp \left[\frac{i}{\hbar} \overrightarrow{\boldsymbol{q}} \cdot \int_{\overrightarrow{\boldsymbol{x}}_{0}}^{\overrightarrow{\boldsymbol{x}}} d \overrightarrow{\boldsymbol{y}}\right] \exp \left\{-\frac{i T}{2 \hbar m} \overrightarrow{\boldsymbol{q}} \cdot \overrightarrow{\boldsymbol{q}}\right\}
$$

Comparing the two equations above, we conclude that

$$
\frac{\hbar^{2}}{2 m} \Delta_{x} K\left(\overrightarrow{\boldsymbol{x}}-\overrightarrow{\boldsymbol{x}}_{0} ; T\right)=i \hbar \frac{\partial}{\partial T} K\left(\overrightarrow{\boldsymbol{x}}-\overrightarrow{\boldsymbol{x}}_{0} ; T\right)
$$

Finally, recalling the relation between the amplitude $K$ and the wave function, namely

$$
\psi(\overrightarrow{\boldsymbol{x}}, t)=\int d^{3} x_{0} K\left(\overrightarrow{\boldsymbol{x}}-\overrightarrow{\boldsymbol{x}}_{0} ; t\right) \psi\left(\overrightarrow{\boldsymbol{x}}_{0}, 0\right),
$$

we also conclude that $\psi(\overrightarrow{\boldsymbol{x}}, t)$ must satisfy the time-dependent Schrödinger equation. At this point, it seems pedagogically instructive to reverse the procedure, and show that the propagation kernel can be determined by solving the diffusion equation (39) which we now assume as given. To this end, we make the following ansatz,

$$
K\left(\overrightarrow{\boldsymbol{x}}-\overrightarrow{\boldsymbol{x}}_{0} ; T\right)=F(T) \exp \left(i M\left(\overrightarrow{\boldsymbol{x}}-\overrightarrow{\boldsymbol{x}}_{0} ; T\right) / \hbar\right)
$$


in terms of two trial functions $F(T)$ and $M\left(\overrightarrow{\boldsymbol{x}}-\overrightarrow{\boldsymbol{x}}_{0} ; T\right)$. The overall normalization constant may be determined by the same boundary condition (34).

In order to determine the form of the trial functions, we demand that the tentative expression (41) satisfies equation (39). Thus, one finds

$$
\frac{F(T)}{2 m} \Delta_{x} M\left(\overrightarrow{\boldsymbol{x}}-\overrightarrow{\boldsymbol{x}}_{0} ; T\right)=-\frac{d F(T)}{d T}
$$

and

$$
\frac{1}{2 m}(\vec{\nabla} M) \cdot(\vec{\nabla} M)=-\frac{\partial M}{\partial T}
$$

Note that equation (43) is just the classical Jacobi equation (23). Thus, without further calculations we can identify the phase of the kernel with the classical action:

$$
M\left(\overrightarrow{\boldsymbol{x}}-\overrightarrow{\boldsymbol{x}}_{0} ; T\right)=S_{\text {cl. }}\left(\overrightarrow{\boldsymbol{x}}-\overrightarrow{\boldsymbol{x}}_{0} ; T\right)=\frac{m}{2 T}\left|\overrightarrow{\boldsymbol{x}}-\overrightarrow{\boldsymbol{x}}_{0}\right|^{2} .
$$

Next, in order to determine the dependence of the kernel pre-factor on $T$, we make use of equation (42). Therefore, we first apply the Laplacian operator to $S_{\text {cl. }}\left(\overrightarrow{\boldsymbol{x}}-\overrightarrow{\boldsymbol{x}}_{0} ; T\right)$ :

$$
\begin{aligned}
& \frac{\partial}{\partial x^{i}} S_{\text {cl. }}\left(\overrightarrow{\boldsymbol{x}}-\overrightarrow{\boldsymbol{x}}_{0} ; T\right)=\frac{m}{T}\left(\overrightarrow{\boldsymbol{x}}-\overrightarrow{\boldsymbol{x}}_{0}\right)_{i} \\
& \frac{\partial^{2}}{\partial x^{k} \partial x^{i}} S_{\mathrm{cl} .}\left(\overrightarrow{\boldsymbol{x}}-\overrightarrow{\boldsymbol{x}}_{0} ; T\right)=\frac{m}{T} \delta_{k i} \\
& \delta^{k i} \frac{\partial^{2}}{\partial x^{k} \partial x^{i}} S_{\mathrm{cl} .}\left(\overrightarrow{\boldsymbol{x}}-\overrightarrow{\boldsymbol{x}}_{0} ; T\right)=\frac{m}{T} \delta^{k}{ }_{k}=\frac{3 m}{T} .
\end{aligned}
$$

Then, we substitute the result into equation (42), which now takes the form

$$
\frac{d F}{d T}=-\frac{3}{2 T} F \Rightarrow \frac{d F}{F}=-\frac{3}{2} \frac{d T}{T} .
$$

Finally, integrating the last equation, we obtain

$$
F(T)=\text { const. } \times T^{-3 / 2} .
$$

Substituting all of the above in the original ansatz (41), leads to the following expression for the propagation kernel,

$$
K\left(\overrightarrow{\boldsymbol{x}}-\overrightarrow{\boldsymbol{x}}_{0} ; T\right)=\frac{N}{T^{3 / 2}} \exp \left(i S_{\mathrm{cl} .}\left(\overrightarrow{\boldsymbol{x}}-\overrightarrow{\boldsymbol{x}}_{0} ; T\right) / \hbar\right) .
$$

Except for the normalization constant which is fixed by the initial condition, this is the same expression obtained from the path integral.

\section{Concluding Remarks}

As argued in Ref. [3], the conceptual foundations of quantum mechanics are still the subject of debate, and thus "it is important to introduce undergraduate physics majors to non-standard developments of quantum mechanics" in the hope that exposure to such developments, early in the curriculum, might motivate some students to focus on the physical principles underlying the theory. In this paper we have taken up that

challenging task by discussing a novel approach to the computation of the sum over 
histories in the path integral.

On the mathematical side, we have suggested a phase space formulation of the Feynman sum over paths in which the spatial trajectory $\overrightarrow{\boldsymbol{y}}(t)$ plays the role of a Lagrange multiplier enforcing the classical equation of motion (18) at the quantum level. Imposing such a constraint, with the aid of a Dirac delta-distribution, reduces the functional integral to an ordinary integral over the arbitrary values of the initial momentum. In the simple case of a free, non relativistic particle, the use of the phase space path integral, or the Lagrangian formulation of it, is largely a matter of choice, in the sense that one arrives at the same expression of the propagation kernel. In the case of relativistic extended systems, the use of the phase space path integral along the same lines discussed here, seems definitely advantageous, if not mandatory. On the physical side, however, our formulation of the path integral reflects a new mechanism of propagation which, in our view, sheds some new light on the connection between classical and quantum systems. The element of novelty can be summarized thus: it is well known that classical mechanics, which is a deterministic theory, demands that one definite value of the initial position and momentum be assigned a priori, and thereafter selects a single classical trajectory that satisfies the equation of motion. On the other hand, in quantum mechanics one cannot fully specify the value of the momentum of a precisely localized particle. The price to pay for specifying the particle position at $\overrightarrow{\boldsymbol{x}}_{0}$ when $t=0$, is that $\overrightarrow{\boldsymbol{p}}$ is completely arbitrary, and therefore one has to sum over all possible values of the threemomentum. This brings into the game the whole family of trajectories which satisfy the classical equations of motion. With all such channels of propagation open, a quantum particle turns to a "wave-like" propagation mode, exploring all of them by "evolving simultaneously" along all classical trajectories corresponding to all possible values and orientations of the momentum, up to the final point $\overrightarrow{\boldsymbol{x}}$ where the particle is detected. Thus, as stated in most textbooks of Quantum Mechanics, but never fully clarified, a quantum particle starts as a pointlike object precisely located at a point, propagates as a wave, only to reappear as a dot on a screen, at the point of detection. We have exploited this dual, wavelike, description of the family of classical trajectories by connecting the quantum evolution of a particle with the Jacobi formulation of Classical Mechanics, i.e., by deriving the diffusion equation from the propagation kernel, and vice versa. The main purpose of the whole exercise was to test the consistency of our approach, and its ability to reproduce some standard results obtained by different methods. Equally important, however, the objective was to clarify, with the familiar example of a free particle, the connection between the classical theory and the central idea upon which the path integral formulation is based, namely, that "trajectories" still play a role in quantum mechanics and that all paths, with their intrinsic randomness, contribute to the evolution of the wave function which is governed by the propagation kernel. Having said that, we may add that our mathematical analysis and its physical interpretation extend well beyond the case of a free, non relativistic particle. Indeed, the very possibility of formulating a quantum mechanics of closed strings and other extended objects[9], points to the effectiveness of our approach to the path integral for studying new fundamental 
physics. By an interesting feedback process, we have found that many new formal and physical properties of our approach to strings can be applied equally well to the case of a point particle which is now regarded as the object of lowest dimensionality in a hierarchy of geometric objects to which the same dynamical principles seem to apply. ¿From the present introduction, the interested reader may eventually progress to a more advanced discussion involving relativistic extended systems, such as strings, membranes and $\mathrm{p}$-branes, with a change in notation rather than substance.

\section{Appendix A. Particle in an External Potential: Short Distance Limit}

The results obtained in the previous sections for a free particle are, of course, exact. However, the same approach can be used to derive the short distance approximation for the particle's kernel in an arbitrary potential $V(\overrightarrow{\boldsymbol{x}})$.

If $\left|\overrightarrow{\boldsymbol{x}}-\overrightarrow{\boldsymbol{x}}_{0}\right|$ is much smaller than the range over which $V(\overrightarrow{\boldsymbol{x}})$ varies significantly, then we can Taylor expand $V(\overrightarrow{\boldsymbol{x}})$ in the neighborhood of $\overrightarrow{\boldsymbol{x}}_{0}$ :

$$
\begin{aligned}
& V(\overrightarrow{\boldsymbol{x}}) \approx V_{0}-\overrightarrow{\boldsymbol{F}} \cdot\left(\overrightarrow{\boldsymbol{x}}-\overrightarrow{\boldsymbol{x}}_{0}\right)+\ldots \\
& \overrightarrow{\boldsymbol{F}}=-[\vec{\nabla} V(\overrightarrow{\boldsymbol{x}})]_{\overrightarrow{\boldsymbol{x}}=\overrightarrow{\boldsymbol{x}}_{0}} .
\end{aligned}
$$

In this case, the path integral reads

$$
\begin{aligned}
& K\left(\overrightarrow{\boldsymbol{x}}-\overrightarrow{\boldsymbol{x}}_{0} ; T\right)=N \int_{\overrightarrow{\boldsymbol{x}}_{0}}^{\overrightarrow{\boldsymbol{x}}}[\mathcal{D} y(t)][\mathcal{D} p(t)] \times \\
& \quad \times \exp \left\{\frac{i}{\hbar}\left[\int_{\overrightarrow{\boldsymbol{x}}_{0}}^{\overrightarrow{\boldsymbol{x}}} d(\overrightarrow{\boldsymbol{p}} \cdot \overrightarrow{\boldsymbol{y}})-\int_{0}^{T} d t \overrightarrow{\boldsymbol{y}}(t) \cdot \frac{d \overrightarrow{\boldsymbol{p}}(t)}{d t}-\int_{0}^{T} d t\left(H-\overrightarrow{\boldsymbol{F}} \cdot\left(\overrightarrow{\boldsymbol{y}}-\overrightarrow{\boldsymbol{y}}_{0}\right)\right)\right]\right\}
\end{aligned}
$$

where $H \equiv H_{0}+V_{0}$. Integration over the particle trajectory gives a "Dirac-delta" whose argument yields the classical equation of motion

$$
\frac{d \overrightarrow{\boldsymbol{p}}}{d t}=\overrightarrow{\boldsymbol{F}}
$$

which leads, in turn, to the expression for the classical momentum

$$
\overrightarrow{\boldsymbol{p}}(t)=\overrightarrow{\boldsymbol{F}} t+\overrightarrow{\boldsymbol{q}}
$$

Thus, the integration over the momentum trajectory can be defined as follows

$$
\int[\mathcal{D} p(t)] \delta\left[\frac{d \overrightarrow{\boldsymbol{p}}}{d t}-\vec{F}\right](\ldots) \equiv \int d^{3} q \int[\mathcal{D} p(t)] \delta[\overrightarrow{\boldsymbol{p}}(t)-\overrightarrow{\boldsymbol{F}} t-\overrightarrow{\boldsymbol{q}}](\ldots)
$$

and the resulting path integral takes the form

$$
\begin{aligned}
K\left(\overrightarrow{\boldsymbol{x}}-\overrightarrow{\boldsymbol{x}}_{0} ; T\right)= & \left(\frac{1}{2 \pi \hbar^{2}}\right)^{3 / 2} \exp \frac{i T}{\hbar}\left(V_{0}-\overrightarrow{\boldsymbol{F}} \cdot \overrightarrow{\boldsymbol{x}}+\frac{\overrightarrow{\boldsymbol{F}}^{2} T^{2}}{6 m}\right) \times \\
& \times \int d^{3} q \exp \left(-\frac{i T \overrightarrow{\boldsymbol{q}}^{2}}{2 \hbar m}\right) \exp \left[-\frac{i}{\hbar} \overrightarrow{\boldsymbol{q}}\left(\overrightarrow{\boldsymbol{x}}-\overrightarrow{\boldsymbol{x}}_{0}+\frac{T^{2}}{2 \hbar m} \overrightarrow{\boldsymbol{F}}\right)\right] \\
& =\left(\frac{m}{2 i \pi \hbar T}\right)^{3 / 2} \exp \frac{i T}{\hbar}\left(V_{0}-\overrightarrow{\boldsymbol{F}} \cdot \overrightarrow{\boldsymbol{x}}+\frac{\overrightarrow{\boldsymbol{F}}^{2} T^{2}}{6 m}\right) \times
\end{aligned}
$$




$$
\times \exp \left[-\frac{i m T}{2 \hbar T}\left(\overrightarrow{\boldsymbol{x}}-\overrightarrow{\boldsymbol{x}}_{0}+\frac{T^{2}}{2 \hbar m} \overrightarrow{\boldsymbol{F}}\right)^{2}\right],
$$

where the normalization constant has been chosen in such a way as to reproduce equation (35) once the potential is switched off.

\section{Appendix B. Three Basic Formulae}

One needs only three basic formulae in order to derive the main results reported in this paper: one is the extension of the Gaussian integral to a $D$-component vector variable, and the other two are representations for the Dirac delta-function.

Generalized Gaussian Integral

$$
\begin{aligned}
\int d \mathbf{X} \exp \left(-\frac{1}{2} X^{i} A_{i j} X^{j}+B^{i} X_{i}\right)= \\
=\text { const. } \times(\operatorname{det} \mathbf{A})^{1 / 2} \exp \left(\overrightarrow{\mathbf{B}} \cdot \mathbf{A}^{-1} \cdot \overrightarrow{\mathbf{B}} / 2\right)
\end{aligned}
$$

where

$$
\mathbf{A}=\left(A_{i j}\right) \quad \text { and } \quad \overrightarrow{\mathbf{B}}=\left(B_{i}\right)
$$

Dirac-delta representations

a) Functional Fourier Transform:

$$
\delta[\overrightarrow{\mathbf{X}}(\lambda)]=(\text { const. }) \times \int[\mathcal{D} P(\lambda)] \exp \left(-i \int d \lambda \overrightarrow{\mathbf{P}}(\lambda) \cdot \overrightarrow{\mathbf{X}}(\lambda)\right)
$$

b) Gaussian Representation:

$$
\delta^{D}(x) \equiv \lim _{\epsilon \rightarrow 0}\left(\frac{1}{\pi \epsilon}\right)^{D / 2} \exp \left(-x^{2} / \epsilon\right)
$$

[1] Some useful annotated references can be found in the Resource Letter by Gutzwiller M C 1998 Am. J. Phys. 66304

[2] Holstein B R 1998 Am. J. Phys 66 583: this article discusses a number of techniques employed in the path integral method, and contains some useful references to the particle propagator in a linear potential

[3] English L Q and Winters R R 1997 Am. J. Phys. 65390

[4] Cohen S M 1997 Am. J. Phys. 66537

[5] Dowrick N J 1997 Eur. J. Phys. 1875

[6] Feynman R P 1948 Rev. Mod. Phys. 20367

[7] Ansoldi S, Aurilia A and Spallucci E 1997 Phys. Rev. D56 2352

[8] Ansoldi S, Aurilia A and Spallucci E 1999 Chaos, Solitons and Fractals "Superstrings, M, F, S ... Theory", 10197

[9] Ansoldi S, Aurilia A and Spallucci E 1996 Phys. Rev. D53 870 Aurilia A, Spallucci E and Vanzetta I 1994 Phys. Rev. D50 6490 Aurilia A, Smailagic A and Spallucci E 1993 Phys. Rev. D47 2536 
[10] Feynman R P and Hibbs A R 1965 Quantum Mechanics and Path Integrals (New York: Mc Graw-Hill)

[11] Dirac P A M 1958 The Principles of Quantum Mechanics, (London: Oxford University Press) Feynman R P, reference [6]. This paper, as well as Dirac's original work can be found reprinted in : Selected Papers on Quantum Electrdynamics (New York: Schwinger J ed., Dover, 1958)

[12] Abbott L F and Wise M 1981 Am. J. Phys. 49 37; see also, Nottale L 1992 Fractal Spacetime and Microphysics (World Scientific)

[13] The path integral approach is discussed in many textbooks besides that by Feynman and Hibbs, reference 10. See, for instance

Felsager B 1981 Geometry, Particles and Fields (Odense: Odense University Press)

Ramond P 1981 Field Theory, A Modern Primer (Reading, Massachusetts: Benjamin/Cummings Publishers)

Ryder L H 1985 Quantum Field Theory (Cambridge: Cambridge University Press)

Schulman L S 1981 Techniques and Applications of Path Integration (New York: Wiley)

Swanson M 1992 Path Integrals and Quantum Processes (New York: Academic Press) 